\title{
Tratamiento ortodóntico oportuno de la clase III en dentición decidua
}

\section{Early treatment of class III malocclusion in primary dentition}

\section{Resumen}

La maloclusión de clase III es la más compleja de diagnosticar y tratar, durante el crecimiento constituye uno de los retos más difíciles con los que se puede encontrar el ortodoncista ya sea en dentición decidua, mixta temprana, mixta tardía o permanente. El objetivo del tratamiento de la maloclusión de clase III en dentición decidua, es lograr un ambiente favorable para un mejor desarrollo dentofacial por ello esta maloclusión debe ser tratada a penas se diagnostica. En el presente artículo se presenta el reporte de un caso, de una paciente de 5 años con 6 meses, con una maloclusión esquelética clase III, resalte negativo, overbite $80 \%$, patrón normodivergente.

Palabras clave: Maloclusión de clase III; tratamiento ortodóncico; dentición decidua.

\section{Abstract}

Class III malocclusion is the most complex to diagnose and treat, during the child's growth is one of the most difficult challenges that the orthodontist can be found either in deciduous dentition, early mixed, late mixed or permanent. The goal of treatment of Class III malocclusion in primary dentition is to achieve a favorable environment for a better dentofacial development so this should be treated just malocclusion is diagnosed. In this article the case of a girl of 5 years 6 months with a skeletal class III malocclusion, negative overjet, $80 \%$ overbite, normodivergent pattern, is reported

Keywords: Class III malocclusion; orthodontic treatment; deciduous dentition.

\section{Caso Clínico}

\section{Angel Delesma-Chumbe ${ }^{1}$, Jennifer Olazabal-Martínez²}

1. Escuela de Pregrado de la Facultad de Odontología de la Universidad Nacional Mayor de San Marcos, Perú.

2. Universidad Nacional Mayor de San Marcos, Perú.

\section{Correspondencia}

C:D: Angel Delesma-Chumbe

Facultad de Odontología de la Universidad Nacional Mayor de San Marcos. Av. Germán Amézaga 375, Lima 1, Perú.

Correo electrónico: afdelesma@hotmail.com

Coautor:

Olazabal: jenita20 @hotmail.com

Recibido: 10-03-15

Aceptado: 12-05-15

\section{Introducción}

Dada la primera clasificación de las maloclusiones por Angle en el año 1899, en la cual describía la Clase III como la posición mesial surco mesiovestibular del primer molar inferior en relación a la cúspide mesiovestibular del primer molar superior y denominada mesioclusión por Lissher, han surgido diferentes clasificaciones no solo teniendo en cuenta únicamente la posición sagital de los primeros molares ${ }^{1,2}$.

Moyers introdujo el concepto de "Síndrome de Clase III", pues añadió a la clasificación de Angle aspectos como la discrepancia en la longitud de arcada, problemas esqueléticos u óseos, disfunciones musculares, problemas dentarios (como mordidas cruzadas anteriores o posteriores, con o sin compensación dentaria) y perfil facial del paciente, donde destaca el aplanamiento de la cresta malar, la deficiencia del tercio medio facial o la prominencia del labio inferior. Consideraba que a pesar de que su origen fuese esquelético, un tratamiento temprano podía redireccionar el crecimiento del paciente y así corregir el síndrome de Clase III ${ }^{3,4,5}$.

La clase III se define como una combinación de cambios esqueléticos y dentoalveolares en los tres planos del espacio: transversal, vertical y sagital, se expresa por retrusión maxilar, prognatismo mandibular, o una combinación de ambas formas ${ }^{6,7}$.

Canut ${ }^{8}$ sostiene la corrección de la clase III tempranamente, tan pronto como sea posible para favorecer precozmente, el normal funcionalismo estomatognatico.

Según Bishara9 ${ }^{9}$ cabe considerar, al evaluar la relación de Clase III, si el problema presenta origen dentoalveolar o esquelético, durante la dentición decidua o mixta. La mordida cruzada anterior puede ser causada por la inclinación inadecuada de los incisivos maxilares y mandibulares, por interferencias oclusales (funcionales) o por discrepancias esqueléticas del maxilar o de la mandíbula. La posibilidad de tratamiento en este momento debe considerarse con cuidado en estos casos.

Junji Sugawara y Mitani ${ }^{10}$ realizaron estudios longitudinales sobre el cambio gradual del crecimiento mandibular y maxilar durante la etapa pre-puberal, puberal y períodos post-puberal, de pacientes con maloclusión Clase III en comparación con sujetos de maloclusion Clase I, encontraron que no mos- traron cambios significativos entre los dos grupos y concluyeron que la maloclusión esquelética Clase III debiese haber establecido antes del período de crecimiento pre-puberal y mantenido después, pero la desarmonía dentoalveolar hace que se vuelva más severa durante este periodo.

Una razón importante para instituir los principios de corrección de la mordida cruzada anterior es evitar las complicaciones asociadas a menudo con él, como la recesión gingival labial de los incisivos inferiores, excesivo desgaste incisal, mayores posibilidades de disfunción de articulación temporomandibular (ATM), un patrón de crecimiento que empeora, estética dental y facial comprometidos, y los efectos psicosociales negativos asociados. Beneficios para el tratamiento temprano pueden incluir: potencial para un cambio ortopédico mayor en un periodo corto de tiempo, mejoras estéticas anteriores en el perfil sonrisa y facial, la prevención de la recesión periodontal y desgaste dental, incrementar la función más temprana, la eliminación de un cambio funcional anterior de la mandíbula, y evitar o disminuir las posibilidades de cirugía ortognática más adelante ${ }^{11}$. 
El diagnóstico ideal de maloclusión Clase III debe ser precoz, tal vez incluso en los dientes deciduos, cuanto antes sea la interceptación plantea importantes efectos ortopédicos más que ortodónticos ${ }^{12}$.

Entre las opciones de tratamiento que disponemos en la literatura, figuran una variedad de opciones, que pueden variar de acuerdo al estadio de maduración ósea y a la edad del paciente, pues no es lo mismo tratar a un paciente en dentición decidua que a uno en dentición permanente, si no se tratan pueden empeorar, acabando en la mayoría de casos con intervenciones de cirugía ortognática una vez se llegue a la edad adulta.

El objetivo del tratamiento temprano consiste en la corrección de las discrepancias esqueléticas, dentoalveolares y musculares, ya sea existentes o en procesos de desarrollo, con el objeto de preparar un mejor entorno orofacial antes de que la erupción de la dentición permanente se haya completado. $\mathrm{Al}$ iniciar las terapias ortodónticas y ortopédicas a una edad más temprana, la necesidad del tratamiento ortodóntico complejo es minimizada, en especial aquella que involucra la extracción de dientes permanentes y la cirugía ortognática ${ }^{13,14}$. Además de regresar al niño a la estética temprana implica contribuir a su autoestima, teniendo en cuenta el aspecto psicológico.

El objetivo de este artículo es brindar una nueva alternativa de tratamiento temprano en pacientes poco colaboradores, con la finalidad de proporcionar un ambiente favorable, para un buen desarrollo funcional del sistema estomatognático, en base a un estímulo funcional masticatorio adecuado.

\section{Reporte del caso}

\section{Diagnóstico y etiología}

Paciente de sexo femenino, de 5 años 6 meses, acude a la consulta acompañada de su madre, el motivo de la consulta fue: "mi hija tiene su mordida al revés".

Antecedentes familiares: ninguno de los familiares directos de la paciente presentan el mismo problema.

Antecedentes clínicos: paciente dolicofacial, asimetría facial, perfil facial cóncavo, tercio inferior ligeramente aumentado, competencia labial, mordida cruzada anterior con un OJ: $-3 \mathrm{~mm}$, $\mathrm{OB}: 80 \%$, mordida invertida anterior con características clínicas de Clase III, modelos de estudio y fotografías extraorales e intraorales llegamos al diagnóstico. (Fig. 1, 2, 3 y 4).

- Diagnóstico: Maloclusión Clase III en dentición decidua.

- Plan de tratamiento con aparatología fija superior con topes de resina para levante de mordida posterior.

- Estrategia del tratamiento consistió en aumento de la dimensión vertical oclusal con los topes de resina en el sector posterior inferior para que la aparatología fija se exprese y se produzca un movimiento de inclinación de los dientes anterosuperiores.

\section{- Tiempo de tratamiento: 3 meses.}

El objetivo principal del tratamiento fue brindarle al paciente un tratamiento ortodóntico temprano para devolver la funcionalidad de la oclusión, corrigiendo la mordida invertida anterior y alteraciones funcionales mejorando así el perfil facial.

El tratamiento consistió en aparatología fija superior con topes de resina inferior para levante de mordida posterior con, tiempo de tratamiento: 3 meses, teniendo en cuenta que la paciente por su corta edad y su temperamento no soportaría un tratamiento prolongado, asimismo se buscó que la aparatología no dependa de la colaboración de la paciente debido a que ella era poco colaboradora, es por ello que optamos por el uso de aparatología fija edgwise, con arco sobreextendido, en una sola cita se instaló brackets en los cuatro incisivos superiores y tubos adhesivos en las segundas molares deciduas con un arco NiTi 0.014 , por 15 días (Fig. 6, 7 y 8), en la segunda cita se observó un leve movimiento de inclinación de los dientes anterosuperiores. Se cambió a un arco sobre extendido con loops en mesial de las segundas molares en acero 0.016 por un mes, con ella se logró una vestibularización ligera de los incisivos anterosuperiores, en una tercera cita se instaló un arco de acero sobre extendido con loops en mesial de las segundas molares en acero 0.018 , por un mes con ello se logró una relación de los incisivos bis a bis, para la cuarta cita, se activaron los loops del arco y se citó en 15 días, luego del cual se observó la corrección de la mordida cruzada, oberjet $1.5 \mathrm{~mm}$, oberbite $2 \mathrm{~mm}$, se retiró la aparatología y se cita en 15 días para evaluación. (Fig. 9, 10, 11 y 12).

\section{Resultados del tratamiento}

Se logró el objetivo de tratamiento en 3 meses, sin necesidad de la colaboración de la paciente para el uso de la aparatología.

\section{Discusión}

El momento de cuando intervenir en el tratamiento para las maloclusiones Clase III ha sido tema de debate a lo largo del tiempo. Desde un tratamiento temprano durante la dentición decidua o mixta primera fase, hasta la erupción completa de todos los dientes o incluso hasta el término del crecimiento ${ }^{11,12}$.

Entre las opciones de tratamiento que disponemos en la literatura, figuran una variedad de opciones, que pueden variar de acuerdo al estado de maduración ósea y a la edad del paciente, si no se tratan pueden empeorar, acabando en la mayoría de casos con intervenciones de cirugía ortognática una vez se llegue a la edad adulta; ante la decisión sobre la opción de tratamiento, existen factores que influyen como son la etiología por la relación causa efecto, el tipo de paciente si este es colaborador, ordenado, cumplido y su entorno familiar el cual también debe estar comprometido con el tratamiento, la mayor parte de las opciones de tratamiento dependen de la colaboración del paciente.

Sadao Sato enfatiza que el plano oclusal es el componente de mayor importancia que afecta al tercio inferior de la cara verticalmente y por ende debe ser modificada con un enfoque clínico adecuado para el tratamiento de la maloclusión Clase III ${ }^{13}$.

Ngan sugiere un tratamiento temprano de la maloclusión Clase III en mordidas cruzadas anteriores, mejorando así la oclusión funcional con la finalidad de eliminar las discrepancias entre la máxima intercuspidación, evitando efectos adversos del crecimiento ${ }^{14}$.

El objetivo del tratamiento temprano consiste en la corrección de las discrepancias esqueléticas, dentoalveolares y musculares, ya sea existentes o en proceso de desarrollo, con el objeto de preparar un mejor entorno orofacial antes de que la erupción de la dentición permanente se haya completado. Al iniciar las terapias ortodónticas y ortopédica a una edad más temprana, la necesidad de tratamiento ortodóntico complejo es minimizada, en especial aquella que involucra la extracción de dientes permanentes y la cirugía ortognática ${ }^{8,9}$. 


\section{FASE DE DIAGNÓSTICO}

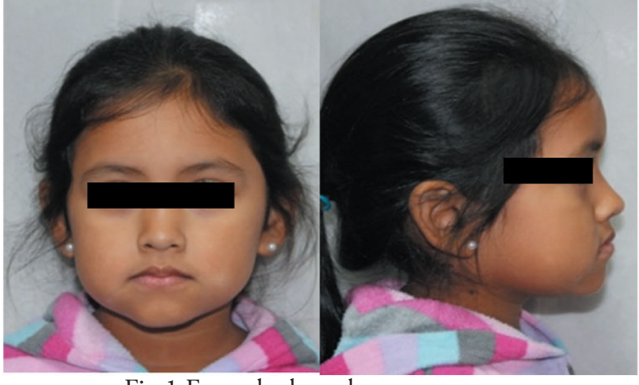

Fig.1 Frontal y lateral

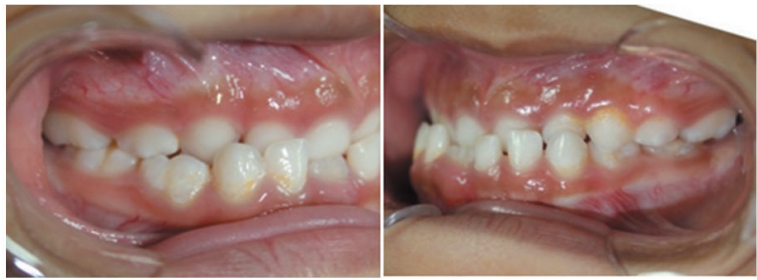

Fig.3. Relaciones molares en Clase III derecha e izquierda

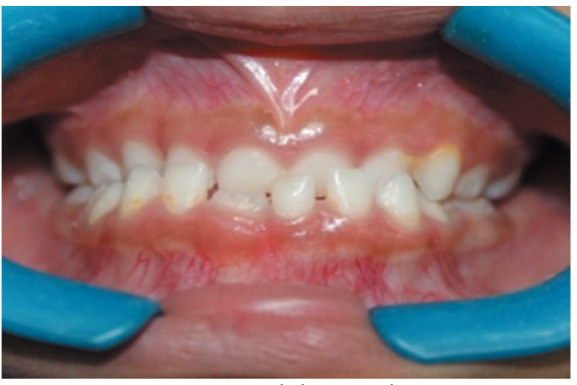

Fig.2. Mordida cruzada anterior

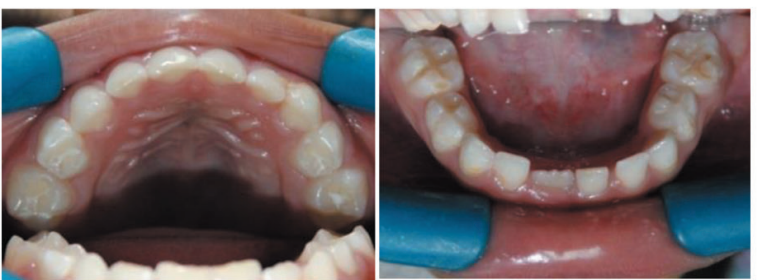

Fig.4. Aspecto oclusal intraoral

TRATAMIENTO A LOS QUINCE DÍAS

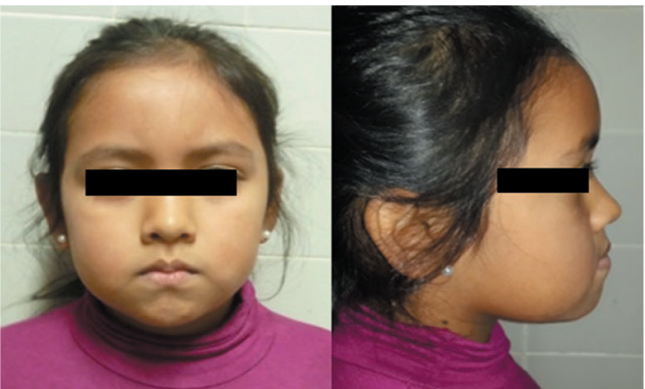

Fig.5. Paciente con tratamiento activo

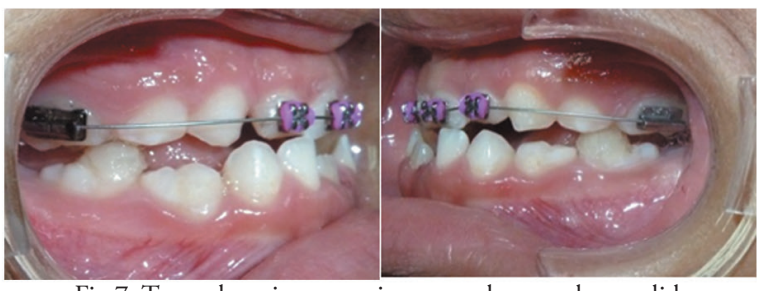

Fig.7. Topes de resina posteriores para levante de mordida

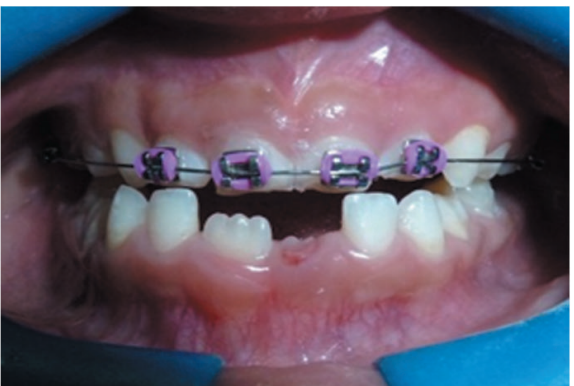

Fig.6. Arco Niti 0.014

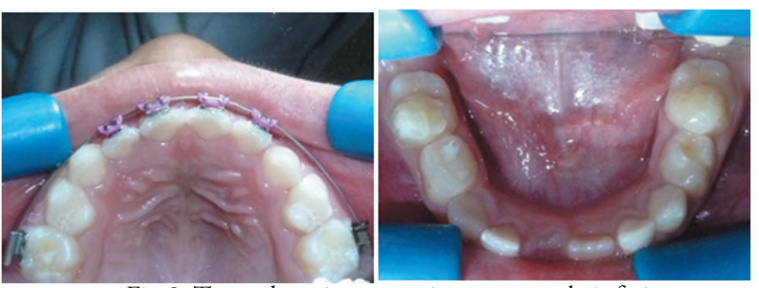

Fig.8. Topes de resina posteriores en arcada inferior

TRATAMIENTO A LOS TRES MESES

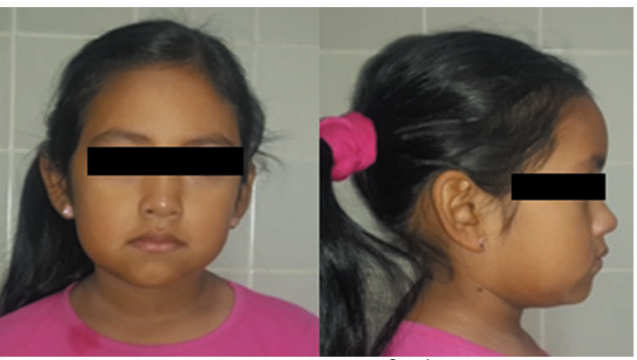

Fig .9. Fase final

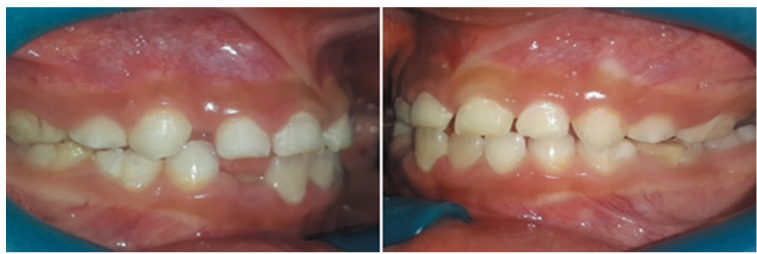

Fig.11.Laterales derecha e izquierda.

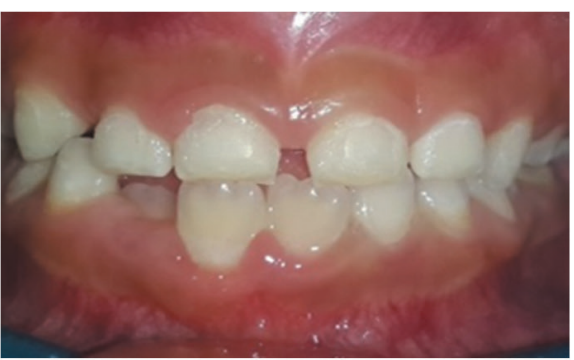

10. Imagen frontal
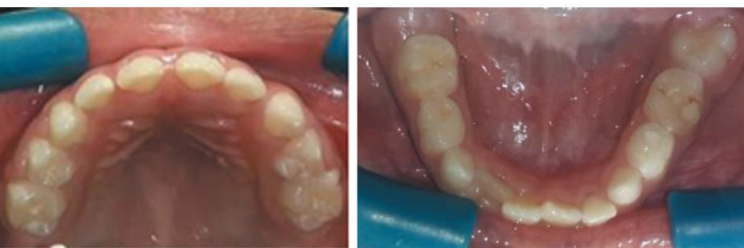

Fig.12. Aspecto oclusal intraoral 
Una razón importante para instituir precozmente la corrección de la mordida cruzada anterior es evitar las complicaciones asociadas a menudo a esta, como la recesión gingival labial de los incisivos inferiores, excesivo desgaste incisal, mayores posibilidades de disfunción de articulación temporomandibular (ATM), un patrón crecimiento que empeora, estética dental y facial comprometidos, y los efectos psicosociales negativos asociados.

Beneficios para el tratamiento temprano pueden incluir: potencial para un cambio ortopédico mayor en un periodo corto de tiempo, mejoras estéticas anteriores en el perfil sonrisa y facial, la prevención de la recesión periodontal y desgaste dental, mejorar función más tempranamente, la eliminación un cambio funcional anterior de la mandíbula, y evitar o disminuir las posibilidades de cirugía ortognática más adelante ${ }^{14}$.

La ortopedia como herramienta de tratamiento temprano ha demostrado ser efectiva en promover cambios favorables de la maloclusión Clase III; sin embargo, 2 factores disminuyen su eficiencia: la necesidad de cooperación del paciente y sus efectos a largo plazo. Además de regresar al niño a la estética temprana implica contribuir a su autoestima, teniendo en cuenta el aspecto psicológico $^{10}$.

\section{Conclusiones}

La literatura nos señala que la maloclusiones Clase III, se instalan desde edades muy tempranas, y que empeoran con el pasar de los años, manifestándose inicialmente con una mordida cruzada anterior, por ende nos recomienda iniciar el tratamiento apenas esta es diagnosticada, en la actualidad contamos con un abanico de posibilidades para su trata- miento, sin embargo la mayoría de ellas requieren de la colaboración del paciente para su uso ya que las aparatologías destinadas a este fin son removibles, es por ello que el uso de la aparatología fija en pacientes poco colaboradores es una alternativa que nos ofrece muchos beneficios para nuestros pacientes, como se vio en el caso presentado, la mordida invertida se corrigió en un corto tiempo y sin la colaboración de la paciente, sin embargo este solo es el inicio del tratamiento, la paciente deberá ser evaluada durante su crecimiento hasta llegar a su término, y requerirá de una segunda fase de tratamiento.

Tenemos que tener presente la recidiva de la mordida cruzada anterior iniciado en el recambio dentario anterior, motivo por el cual en este caso se habló con los padres de la niña, explicándoles que esta solo es la primera fase del tratamiento, y que requeriremos continuar evaluándola durante todo su crecimiento.

\section{Referencias bibliográficas}

1. Angle E. Classification of Malocclusion. The Dental Cosmos 1899;41:248-64

2. Soldevilla LC. Corrección de mordida cruzada anterior y posterior bilateral en dentición decidua con aparatología removible adhesiva. Reporte de un caso. Odontol. Sanmarquina 2004; 8(2):49-53.

3. Canut Brusola JA. Ortodoncia Clínica y Terapéutica. $2^{\text {a }}$ ed. Barcelona: Masson; 2001.

4. Bishara SE. Ortodoncia. $1^{\mathrm{a}}$ ed. Mexico: Mc.Graw Hill; 2003.

5. Sugawara J, Mitani H. Facial growth of skeletal Class III malocclusion and the effects, limitations, and longterm dentofacial adaptations to chin cap therapy. Seminars in orthodontics.1997; 3(4):244-254.

6. Patrick KT. Managing the developing Class III malocclusion with palatal expansion and facemask therapy. Am J Orthod Dent.2002;122(1):349-352

7. Kapust AJ, Sinclair PM, Turley PK. Cephalometric effects of face mask/expansion therapy in Class III children: a comparison of three age groups. Am J Orthod Dent.1998;113(2):204-212

8. Mc Namara J; Brudon W. Tratamiento Ortodoncico y Ortopédico en la Dentición Mixta. USA: An Arbor, Needham Press; 1995.

9. Ellis E, Mc Namara JR. Components of adult Class III malocclusion. J Oral Maxillofac Surg. 1984; 42(5):295-305

10. Saadia M, Torres E. Sagittal changes after maxillary protraction with expansion in Class III patients in the primary, mixed and late mixed dentitions: A longitudinal retrospective study. Am J Orthod Dentofacial. 2000;117(6),669-68.

11. Chul Jang J. Controversies in the timing of orthodontic treatment. Semin Orthod.2005;11:112-8

12. Gianelly AA. One phase versus two phase treatment. Am J Orthod Dentofac Orthop.1995;108:558-9

13. Sadao Sato. Case Report: Developmental characterization of skeletal Class III malocclusion. The Angle Orthodontist. 1994;64(2):105111.

14. Ngan P. Early treatment of class III malocclusion:is it worth the burden? Am J Orthod Dentofac Orthop. 2006;129(4):82-5 